Background A high-quality screening colonoscopy is critical in the prevention and early detection of colorectal cancer. Hence, several innovations and techniques have been proposed to potentially improve the quality of colonoscopy. One of which is the use of new generation high definition colonoscopes to increase the adenoma detection rate (ADR). This study aims to determine the difference of ADR between the new generation high definition colonoscopes.

Methods This is a single-centre, retrospective cross-sectional study that compares the ADR with screening colonoscopies using 180/190 series Olympus colonoscopes and 290 series Olympus colonoscopes. A review of endoscopy databank over a 6 month period (January 2019 to June 2019) was done. Asymptomatic patients, age 50 - 75 years, classified as average risk for developing colon cancer, who had their indexed colonoscopy performed by experienced endoscopist were included. Results A total of 197 out of 340 patients reviewed met the inclusion criteria. 64\% of colonoscopies were performed using the 290 series Olympus colonoscopes. The majority of included patients are male, $77 \%$ of patients in the $180 / 190$ series group and $53 \%$ of patients in the 290 series group. The ADR using the $180 / 190$ series Olympus colonoscope group is $25 \%$ (95\% CI of $17 \%-37 \%$ ) as compared to $45 \%$ (95\% CI of 27\%-54\%) using the 290 series Olympus colonoscopes. The difference is statistically significant with a p-value of 0.006 .

Conclusions The 290 series Olympus colonoscopes showed a statistically significant higher adenoma detection rate as compared to the $180 / 190$ series Olympus colonoscopes. This reflects a significant difference between the new generation high definition colonoscopes in terms of ADR.

\section{IDDF2020-ABS-0185 PHARMACOECONOMIC EVALUATION OF BEVACIZUMAB OR CETUXIMAB COMBINED CHEMOTHERAPY FOR ADVANCED COLORECTAL CANCER IN REAL WORLD}

Yunqi Hua*, Chenlin Wang, Cuiyun Zhao, Kun Cao, Yunjian Jin, Xi Li, Yaqin Tan, Min Li, Meili Zhao. Baotou Tumor Hospital, Clinical Oncology of Baotou Medical College, China

\subsection{6/gutjnl-2020-IDDF.110}

Background Clinical first-line data are needed to support the economic evaluation of drugs in the real world. We will see the efficacy of two targeted drugs (Bevacizumab versus Cetuximab) combined with chemotherapy for advanced colorectal cancer and the economics of evaluating two targeted drugs in real-world.

Methods Between 2018 and 2020,68 patients were treated with targeted drugs (Bevacizumab versus Cetuximab) combined with chemotherapy. All patients met the diagnostic requirements of advanced colorectal cancer. 1. Cases were divided into 2 groups: Group A received bevacizumab plus chemotherapy(40cases), and Group B received Cetuximab plus chemotherapy(28cases). 2. Treatment programme: Group A: Bevacizumab $5 \mathrm{mg} / \mathrm{Kg}$ combination chemotherapy (FOLFOX/ FOLFIRI); Group B: K-ras Gene Wild type colorectal cancer patients, Cetuximab $400 \mathrm{mg} / \mathrm{m}^{2}$ in the first week, then 250 $\mathrm{mg} / \mathrm{m}^{2}$ per week, Combined chemotherapy (FOLFOX/FOLFIRI), 14 days for 1 cycle. Evaluation of efficacy and economics after 4 cycles. 3. The establishment of cost: including: drug costs, bed costs, inspection costs, treatment costs, nursing costs. The total cost is the sum of the above costs. 4. The short-term curative effect evaluation is based on RECIST. Cost-effectiveness ratio $(\mathrm{c} / \mathrm{e})=$ efficiency/total cost. 5. Statistical Methods All data were analyzed by SPSS 17.0. statistical software; $P<0.05$ was statistically significant.

Results The clinical effective rate of Bevacizumab combined with chemotherapy was (12/40)30.0\%. The clinical effective rate of Cetuximab combined with chemotherapy was $(8 / 28)$ $28.6 \%$, there was no significant difference between the two groups $(P>0.05)$ The average cost of Bevacizumab combined chemotherapy group was 11,500 yuan, and that of Cetuximab group was 29,800 yuan, the difference was statistically significant $(P<0.05)$; The cost-effectiveness ratio was $(0.3 /$ $1.15) 26.09 \%$ in Bevacizumab combined chemotherapy group and $(0.28 / 2.98) 9.40 \%$ in Cetuximab combined chemotherapy group, the difference was statistically significant $(P<0.05)$.

Conclusions In the real world, Bevacizumab combined chemotherapy and Cetuximab combined chemotherapy have the same clinical efficacy. The former is better in economic evaluation, therefore, Bevacizumab combined with chemotherapy is the first choice for patients with advanced colorectal cancer.

\section{IDDF2020-ABS-0186 THE EFFECT OF PROBIOTICS IN DIARRHEA AMONG ADULT HIV PATIENTS: A SYSTEMATIC REVIEW AND META- ANALYSIS}

${ }^{1}$ Elisa Francesca Mendoza*, ${ }^{1}$ Ferdinand Santoceldes, ${ }^{2}$ Marie Antoinette Lontok, ${ }^{2}$ Nikko Theodore Raymundo. 'Department of Medicine, St. Luke's Medical Center Global City, Philippines; ${ }^{2}$ Institute of Digestive and Liver Diseases, St. Luke's Medical Center Global City, Philippines

\subsection{6/gutjnl-2020-IDDF.111}

Background Diarrhea is one of the most common gastrointestinal symptoms in human immunodeficiency virus (HIV) infection. It is associated with significant morbidity and mortality. Probiotics have been studied as an alternative and adjunct treatment for diarrhea in children. Selected Lactobacillus strains have shown to reduce the severity and duration of diarrhea. However, there are no studies on the effect of probiotic use in HIV patients with diarrhea. This study analyzes the effect of probiotics on the immune function of HIV patients with diarrhea.

Methods A comprehensive, computerized literature search from PubMed Central, Embase, Cochrane Library, and OVID was performed with the following search terms: probiotics, human immunodeficiency virus, acquired immunodeficiency disease, diarrhea and gastroenteritis. Two randomized controlled trials (RCT) were selected and validated using GRADE criteria. Trial results were combined under a random-effects model. The Cochrane Review Manager Software version 5.3 was used for all analyses.

Results Two RCT comprising 127 patients were analyzed. In the random-effects model, the pooled odds ratio (OR) of diarrhea was 0.53 ; $95 \%$ CI $(0.16-1.80)$ with homogeneity at $\mathrm{I}^{2}=$ $41 \%$. The pooled data of the two studies showed a trend towards a beneficial effect of probiotics and resolution of diarrhea but did not show a protective effect of the probiotic.

Conclusions There is no clear association on the effect of probiotics and diarrhea in HIV patients. This meta-analysis confirms the need for further studies on examining the relationship of probiotics and HIV-related diarrhea. 